analysis of morphometric data. But this finding is tempered by the fact that Oxnard's analysis was conducted only at the generic level and therefore missed the major differences that exist between individual species within genera. Field data have revealed, for example, that there are important locomotor differences between species of the genus Galago.

There is no doubt that this book represents a significant new contribution to the literature on primate locomotion. At the same time, it must be emphasized that even this combined approach has its limitations. The various clustering procedures presented for both morphometrics and niche metrics are simply methods of data presentation. In themselves, they do not lead us any further along the road to analysis and interpretation. It is difficult to see, for instance, how the approach presented can be applied to the interpretation of fossil primates; the morphometric analyses are too abstract and are confined to the generic level, while niche data are simply not available at the requisite level of refinement for fossil species. It is, indeed, my personal conviction that multivariate studies of prosimian locomotion will never lead to real understanding until the influence of body size has been explicitly taken into account. In this book, the influence of body size is not even mentioned until page 124 , where it is then stated that this factor "cries out" for analysis. It is therefore all the more surprising that the authors have virtually omitted any mention of the abundant recent literature on allometric scaling of locomotor and dietary variables in primates.

The basic point is this: if we compare (say) locomotor patterns of a smallbodied arboreal macaque with those of a larger-bodied terrestrial macaque and find differences, to what extent do these differences reflect a real divergence between arboreal and terrestrial locomotor adaptation and to what extent do they merely reflect the effect of body size? The problem can only be tackled by restricting comparisons to species of the same body size or by applying an appropriate procedure to 'remove' the size effect. My own interest in this question was in its infancy when I took part in a conference at the

\section{The Evolution of Homo erectus (Correction)} In Michael Day's review of The Evolution of Homo erectus by G. Philip Rightmire (Nature 348,$688 ; 1990$ ) the sentence 'This group of material formed the basis for Louis Leakey's theory that man arose as an Asian species rather than one from Africa', should have read 'It was upon this group of material that was based the theory that man arose as an Asian species rather than one from Africa as believed by Louis Leakey'. This editorial error, for which we apologize, reversed the author's intended meaning; Louis Leakey never believed man to be an Asian species and always favoured an African origin.
Zoological Society of London in 1972. After Oxnard had presented his paper on morphometrics, the late Nigel Barnicot asked him to explain how he dealt with the confounding influence of body size. Oxnard's answer was that he believed that his results had not been heavily biased by size effects. One of the major conclusions to emerge from Animal Lifestyles and Anatomies, unheralded by the authors, is that this reply was probably in error. The multivariate pattern that emerges from niche metrics is convincingly shown to be influenced by body size and that pattern is then shown to be closely similar to that obtained from Oxnard's multivariate analysis of morphometric data. It therefore presumably follows that the latter is also influenced by body size (QED). Perhaps when morphometrics and niche metrics have been joined by allometrics we will finally achieve the understanding of primate locomotor adaptation that we are all seeking.

R. D. Martin is at the Anthropological Institute and Museum, University of Zurich, Winterthurerstrasse 190, CH-8057, Zürich, Switzerland.

\section{To boldly go . . .}

\section{Nicole Borderies}

Uranus the Planet, Rings and Satellites. By Ellis D. Miner. Horwood: 1990. Pp. 334. $£ 35.50$.

THE Voyager missions to the outer planets of the Solar System were characterized by the ability of the Jet Propulsion Laboratory (JPL) engineers to respond successfully to all the challenges that they faced, and by the wealth of scientific data obtained during each encounter. The Voyager spacecraft had been designed to fly by the systems of Jupiter and Saturn. Only after the successful encounter of Voyager 1 with Saturn and Titan, were Voyager project personnel given permission by NASA Headquarters to target Voyager 2 for a Uranus trajectory. Voyager 2 flew by Uranus on 24 January 1986 and continued on to an encounter with Neptune on 25 August 1989, 12 years after its launch.

Ellis Miner, who has served as Assistant Project Scientist since shortly after the launches of Voyagers 1 and 2 in the late summer of 1977 , presents an insider's point of view of these missions. This, together with his goal of comprehensiveness and the historical perspective he adopts, are the strongest characteristics of this book. Remarking that the extraordinary successes of the Voyager missions cannot be attributed to good luck, Miner recalls how the Voyager missions came to life. In 1966, Gary Flandro, a JPL and Caltech scientist, showed that gravity assist (the phenomenon by which a spacecraft passing a planet in certain conditions increases its velocity) could be used to send a single spacecraft to several planets of the outer Solar System. In 1970, JPL proposed a 'Grand Tour' mission to NASA, which involved sending two spacecraft to Jupiter, Saturn and Pluto, followed by two more spacecraft to Jupiter, Saturn and Uranus. In response to a request from NASA headquarters for a less expensive version of the 'Grand Tour', JPL proposed a two-spacecraft Mariner Jupiter-Saturn (MJS) mission. Because the MJS spacecraft was developing into something quite different from the previous Mariner spacecraft, it was called Voyager.

Miner's insider view also puts him in a good position to describe the technical aspects of the missions. These include for instance: the instrumentation for the 11 scientific investigations; the launches of the two spacecraft; the problems along the way; the enhancement of the capability of the Deep Space Network to detect Voyager's signal from Uranus; the reprogramming of the computers on board Voyager 2. Another advantage of Miner's unique involvement in the Voyager project is that it gives him a deep understanding of how the project was managed. He explains how the major scientific goals of the Uranus encounter were selected and how the planning was done. He even shows organization charts with the names of the people involved in the project at the time of the encounter with Uranus.

Miner's book is also about science. Here again, his goal is to be comprehensive. The discovery of Uranus by Sir William Herschel is recounted, as well as the discovery of the satellites, and more recently that of the rings by J. L. Elliot and his colleagues. Our pre-Voyager knowledge of the system of Uranus, its rings and its satellites, is described towards the beginning of the book, whereas the state of knowledge after Voyager is given in the last chapters. This information is classically divided into 'Interior', 'Atmosphere', 'Magnetosphere', 'Rings' and 'Satellites'. The description of the new data obtained by Voyager is fairly detailed. Due to the intent of the author to write a book understandable by non-scientists and to the fact that the detailed study of the Voyager data has not been completed, the parts on the interpretation of the data have a more sketchy and preliminary character.

I enjoyed reading the book and I think it would appeal to a wide range of readers who would like to have a feel of what it is to be a part of space exploration.

Nicole Borderies has recently moved to the Jet Propulsion Laboratory, California Institute of Technology, 4800 Oak Grove Drive, Pasadena, California 91109, USA. 